\title{
Identification of a non-linear landing gear model using nature-inspired optimization
}

\author{
Felipe A.C. Viana ${ }^{a}$, Valder Steffen Jr. ${ }^{a}$, , Marcelo A.X. Zanini ${ }^{\mathrm{b}}$, Sandro A. Magalhães ${ }^{\mathrm{b}}$ and \\ Luiz C.S. Góes ${ }^{\mathrm{c}}$ \\ ${ }^{a}$ School of Mechanical Engineering, Federal University of Uberlândia, Av. João Naves de Ávila 2121, Campus \\ Santa Mônica, 38400-902, Uberlândia, MG, Brazil \\ ${ }^{\mathrm{b}}$ Embraer - Empresa Brasileira de Aeronautica S.A., Av. Brigadeiro Faria Lima 2170, 12227-901, S ão José dos \\ Campos, SP, Brazil \\ ${ }^{\mathrm{c}}$ Department of Mechanical-Aeronautical Engineering, Instituto Tecnol ógico de Aeronáutica, Praça Marechal \\ Eduardo Gomes 50, 12228-900, Sao José dos Campos, SP, Brazil
}

\section{Received 2007}

Revised 2007

\begin{abstract}
This work deals with the application of a nature-inspired optimization technique to solve an inverse problem represented by the identification of an aircraft landing gear model. The model is described in terms of the landing gear geometry, internal volumes and areas, shock absorber travel, tire type, and gas and oil characteristics of the shock absorber. The solution to this inverse problem can be obtained by using classical gradient-based optimization methods. However, this is a difficult task due to the existence of local minima in the design space and the requirement of an initial guess. These aspects have motivated the authors to explore a nature-inspired approach using a method known as LifeCycle Model. In the present formulation two nature-based methods, namely the Genetic Algorithms and the Particle Swarm Optimization were used. An optimization problem is formulated in which the objective function represents the difference between the measured characteristics of the system and its model counterpart. The polytropic coefficient of the gas and the damping parameter of the shock absorber are assumed as being unknown: they are considered as design variables. As an illustration, experimental drop test data, obtained under zero horizontal speed, were used in the non-linear landing gear model updating of a small aircraft.
\end{abstract}

Keywords: Inverse problems in dynamics, model updating of a landing gear, nature-inspired optimization techniques, particle swarm optimization, genetic algorithms

\section{Introduction}

The current state-of-the-art in the design of landing gears embraces the use of modern computer-based methodologies (Computer-Aided Design and Computer-Aided Engineering (CAD/CAE), instead of a time consuming process of prototype trial and error [1,2]. By using a landing gear model, different configurations can be tested before proceeding to the construction of the physical model. This approach has greatly improved the speed and quality of the landing gear design process and has become an essential methodology of landing gear design. In the CAD/CAE context, model updating is an important step, since the identified model becomes an effective tool to analyze and predict the dynamics of the system under different operating conditions.

The present contribution reports the aircraft landing gear model update by using a nature-inspired optimization technique, namely the LifeCycle Model [3]. The landing gear model is described in terms of geometry, internal

\footnotetext{
${ }^{*}$ Corresponding author. Tel.: +55 343239 4148; Fax: +55 343239 4206; E-mail: vsteffen@ mecanica.ufu.br.
} 
volumes and areas, shock absorber travel, tire type, and gas and oil characteristics of the shock absorber. However, this problem has been historically time consuming, i.e., many iterations are needed before obtaining a good match between experimental testing and analytical modeling. In this scenario, some difficulties that are intrinsic to inverse problems may arise:

- an accurate mathematical model of the system is required, since the results of the identification procedure rely upon the mathematical model used;

- in general, identification problems are ill conditioned from the mathematical point of view. This means that the procedure is sensitive to noise that can contaminate experimental data; and

- experimental data are incomplete either in the spatial sense (responses are available only in a limited number of positions along the structure), as in the time sense (responses are obtained in a given time interval). Consequently, the uniqueness of the solution cannot be assured.

It is well known that the solution of inverse problems by using classical gradient-based optimization methods is a difficult task due to the existence of local minima in the design space. Moreover, such methods require an initial guess of the solution and it is not possible to assure global convergence. The literature reports cases successfully solved by using different heuristic methods. As an example, in Kristinsson and Dumont [4] Genetic Algorithms (GA) are used to estimate discrete and continuous design variables. The results obtained employing this algorithm are considered to be well suited to the adaptive control problem in combination with a pole placement scheme utilizing the knowledge of poles and zeros. Rojas et al. in [5] presented an inverse procedure for the determination of external loads as an optimization problem solved by Particle Swarm Optimization (PSO), given the dynamic responses of the loaded structure and its corresponding finite element model.

All these aspects and successful applications have motivated the authors of this paper to explore a hybrid approach as a tool for the parameter identification of a non-linear landing gear model. This optimization technique is based on two heuristic methods, namely GA and PSO combined in a strategy known as LifeCycle Model (LC), which was introduced by Krink and Løvberg in [3].

An optimization problem is formulated so that the objective function represents the difference between the measured characteristics of the system and its model counterpart. The polytropic coefficient of the gas and the damping parameter for the shock absorber compression are assumed as being unknown: they are considered as design variables. The polytropic coefficient depends on the operation speed and heat transfer rates within the landing gear. In addition, the fact that many gears use oil in contact with gas instead of using a separator piston makes this prediction even harder due to the air/oil mixture operation. The damping parameter is sensitive to the following characteristics of the system: the geometry of the orifices of the throttle valve, the flow condition at the both downstream and upstream faces of the throttle valve, the hydraulic fluid properties and manufacturing conditions. Some of these characteristics are difficult to predict and the development of the theoretical model is very time-consuming.

Besides the two mentioned unknown parameters, other ones, such as the damping parameter for the shock absorber extension and the tire stiffness, could also be considered as unknowns in the identification procedure. However, no particular identification process was applied to them. The damping parameter for the shock absorber extension has no influence in the main focus of the proposed landing gear mathematical model, which is the prediction of the maximum loads transferred to aircraft and the maximum deflections of the shock absorber and tire. In case of the tire stiffness, the values used in the theoretical model were obtained from experimental tests performed by the tire manufacturer. Thus, it is not expected relevant variation between the tire stiffness used in the model and those obtained in the drop test.

In order to show the optimization strategy and the effectiveness of the LC approach, experimental drop test data, obtained under zero horizontal speed, were used in the non-linear landing gear model updating of a small aircraft.

\section{Drop test arrangements}

During the certification process of commercial aircrafts, the so-called drop tests are used to access the energy absorption properties of landing gears. In this type of test, the landing gear falls freely from a specified height above 


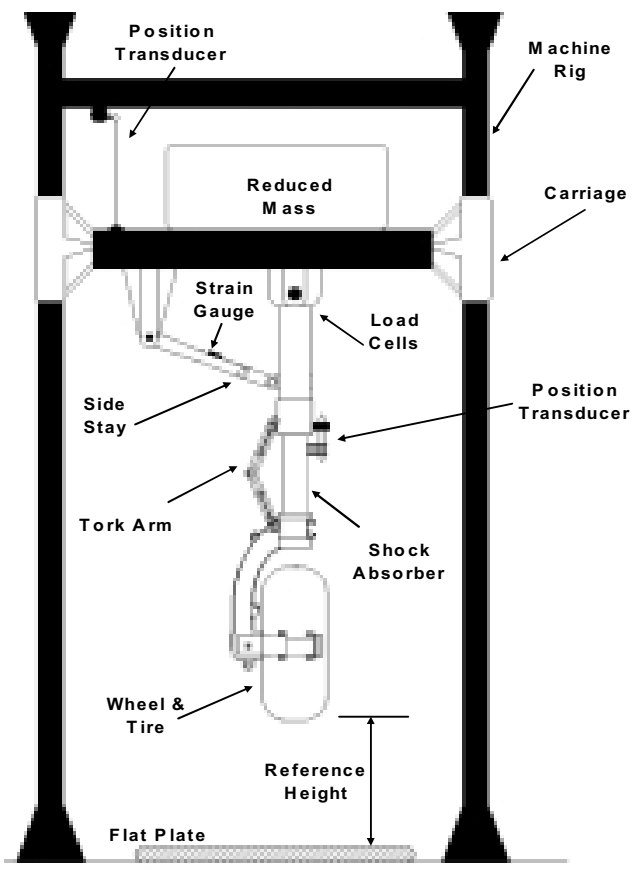

(a) Drop test sketch.

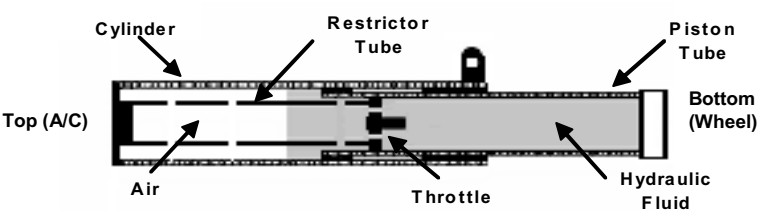

(b) Oil-pneumatic shock absorber.

Fig. 1. Drop test arrangement.

the ground. Considering the category of transport airplanes, at least the vertical load factor (ratio between dynamic and static load) must be validated through drop tests. Besides, the drop test campaign is important for a set of reasons, such as:

- determining the design loads,

- verifying the energy absorption capabilities, and

- updating the landing gear computational model, which can be used in future design cycles.

Figure 1(a) presents a simplified scheme of the drop test facility and the landing gear components. It can be observed that the landing gear is attached to a carriage that runs vertically between two rigs. This carriage is pre-loaded with the equivalent aircraft reduced mass, which represents the portion of the aircraft mass supported by the landing gear in landing impact. The total amount of potential energy used during the test can be obtained by the initial position from where the carriage falls. The lower chamber contains hydraulic fluid that is forced to flow through small orifices (throttle) as a result of the piston tube displacement during the landing operation. Part of the impact energy is dissipated in this process and the air contained in the upper chamber is compressed. After the maximum deflection of the shock absorber, part of the hydraulic fluid flows back into the lower chamber due to the expansion of the compressed air. In this extension stage, the number of orifices through which the fluid flows is such that the contact between the tire and the runway is guaranteed, thus avoiding the rebound effect. The shock absorber considered in the present work uses eight orifices for compression and four orifices for extension. Position transducers are used to determine the vertical displacement of the carriage and the deflection of the shock absorber. The difference between these two parameters gives the tire deflection. Three-dimensional load cells together with strain gages are used to obtain the impact loads transferred to the aircraft. The aerodynamic lift is disregarded in the present contribution. Finally, the landing gear with an oil-pneumatic shock absorber is adopted as a reference, for illustration purposes.

In a realistic scenario, the set of values for the reduced mass is obtained according to the information about the aircraft attitude at touch down. In the so-called "two-points landing" attitude, the aircraft can be in either a level 
or tail down attitude. In this case, the main landing gears touch the ground first and, therefore, the reduced mass is obtained by distributing the aircraft mass between both the main landing gears. In a "three-points landing" all landing gears contact the ground at the same time and, consequently, the reduced mass is obtained by distributing the aircraft mass among all the landing gears. Specifically for "three-points landing", the reduced mass is calculated by considering a longitudinal load factor, as defined by proper design requirements for representing the effects of the aircraft angular acceleration at the instant of the landing impact in the nose landing gear reduced mass. For example, the airworthiness standards for certification of transport category aircrafts in North America, FAR Part-25, state that the nose landing gear reduced mass must be calculated using a longitudinal load factor of 0.25 . The test height is obtained by equaling the kinetic energy at the instant the tire touches the ground to the potential energy. The desired vertical velocity at the instant of the landing impact is considered as a reference to calculate the kinetic energy. The potential energy is obtained by the initial position of the carriage before the test. The following formula shows that the test height is independent of the mass:

$$
h=\sqrt{\frac{v^{2}}{2 \cdot g}},
$$

where $h$ is test height, $v$ is the vertical velocity and $g$ corresponds to the acceleration of gravity.

In a drop test campaign, the landing gear is submitted to different experimental configurations for both reduced mass and initial position, in order to cover the maximum impact energy expected in the aircraft operational life. When the aerodynamic lift is not represented in the drop test machine by a hydraulic actuator, it is necessary to correct the reduced mass in order to compensate for the effect of lift. In this case, using an effective mass that is obtained according to the airworthiness requirements, the drop test is performed.

The tests are made using an instrumented landing gear for which variables such as internal pressure, outside temperature, system load and displacement are recorded during the impact. Position transducers are used to determine the vertical displacement of the carriage and the deflection of the shock absorber. The difference between these parameters gives the tire deflection. Three-dimensional load cells at the landing gear main attachments and strain gauge at the landing gear side stay are used to obtain the impact loads transferred to the aircraft. The total vertical load transferred to the reduced mass is obtained by adding the vertical load at each main attachment to the vertical component of the side stay load. The vertical and longitudinal loads can be also measured at the flat plate where the gear touches down. With exception to the inertial loads from the acceleration of the "unsprung" mass, the resultant loads at the flat plate and at the attachments should cancel each other. In the analyzed test rig, the flat plate was not instrumented.

\section{Non-linear landing gear model}

This section gives details regarding the non-linear modeling of the landing gear. The obtained model allows the identification of the studied parameters using a white-box formulation for the identification problem. The model for the studied drop test system must be capable of predicting the vertical loads transferred to the reduced mass and the deflections of the shock absorber and tire. It is worth mentioning that the developed model does not intend predicting the horizontal drag loads due to friction between the tire and the ground.

Assuming the drop test machine as being infinitely rigid and that the landing gear is attached to the reduced mass, the two degree of freedom analytical model presented in Fig. 2(a) is used to describe the vertical displacements $Z_{1}$ (machine carriage) and $Z_{2}$ ("unsprung" mass vertical displacement or tire deflection) of the reduced mass $M_{1}$ and "unsprung" mass $M_{2}$, respectively. The shock absorber deflection $Z$ is determined by the difference between the displacements $Z_{1}$ and $Z_{2}$. As can be observed, the proposed model is limited to the cases where the landing gear has no inclination angles, i.e. the landing gear is vertically installed in the aircraft. This restriction may be overcome by using trigonometric relations to calculate the vertical displacement $Z_{1}$ and by adding the effects of the shock absorber friction of the bearings.

A spring and a damper represent the shock absorber in the analytical model. Both of them will be first treated as linear components, with coefficients $K_{1}$ and $b$, respectively, in order to develop the differential equations that describe the dynamic behavior of the landing gear. The non-linear effects regarding the gas-oil compression and the 
hydraulic resistance of the orifices will be included in the final differential equations. This approach is also valid for the tire, which is represented in the analytical model by a spring whose stiffness constant is given by $K_{2}$.

Besides the hydraulic resistance and gas-oil compression effects, the damping due to the shock absorber bearing friction force is also relevant in the dynamic behavior of the landing gear [6]. As the hydraulic fluid used in the oil-pneumatic shock absorbers have poor lubricating properties, the bearing friction force can be modeled as a dry friction where the normal forces between the bearings and the sliding cylinder walls are multiplied by a friction coefficient. For drop tests conditions, where no longitudinal loads at the wheel axle is generated and the landing gear has not inclination angle, as assumed in the proposed model, the normal force between the bearings and the sliding cylinder walls can be neglected for a first approach and, consequently, no bearing friction forces are produced.

Lagrange's equations are applied to the analytical model to derive the equations of motion. Initially, the Lagrangian is expressed in terms of both reduced and "unsprung" masses vertical displacements, $Z_{1}$ and $Z_{2}$, respectively, that are the generalized coordinates of the system. The only non-conservative force is the hydraulic resistance of the orifices that is expressed in terms of the Rayleigh's dissipation function. No external generalized forces associated with the generalized coordinates are taken into account.

Thus, the equations of motion are given by:

$$
\left\{\begin{array}{l}
\frac{d}{d t}\left(\frac{\partial L}{\partial \dot{Z}_{1}}\right)-\frac{\partial L}{\partial Z_{1}}+\frac{\partial R}{\partial \dot{Z}_{1}}=0 \\
\frac{d}{d t}\left(\frac{\partial L}{\partial \dot{Z}_{2}}\right)-\frac{\partial L}{\partial Z_{2}}+\frac{\partial R}{\partial \dot{Z}_{2}}=0 .
\end{array}\right.
$$

The Lagrangian, $L$, is defined by the difference between the kinetic energy, $T$, and the potential energy, $V$. The Rayleigh's dissipation function, $R$, is given by:

$$
\begin{aligned}
& L=\frac{M_{1} \cdot\left(\dot{Z}_{1}\right)^{2}}{2}+\frac{M_{2} \cdot\left(\dot{Z}_{2}\right)^{2}}{2}-M_{1} \cdot g \cdot Z_{1}-M_{2} \cdot g \cdot Z_{2}-\frac{K_{1} \cdot\left(Z_{1}-Z_{2}\right)^{2}}{2}-\frac{K_{2} \cdot\left(Z_{2}\right)^{2}}{2} \\
& R=\frac{b \cdot\left(\dot{Z}_{1}-\dot{Z}_{2}\right)^{2}}{2} .
\end{aligned}
$$

From Eqs (2) to (4), the landing gear dynamic behavior can be described by the following differential equations:

$$
\left\{\begin{array}{l}
M_{1} \cdot \ddot{Z}_{1}+K_{1} \cdot\left(Z_{1}-Z_{2}\right)+b \cdot\left(\dot{Z}_{1}-\dot{Z}_{2}\right)=M_{1} \cdot g, \\
M_{2} \cdot \ddot{Z}_{2}-K_{1} \cdot\left(Z_{1}-Z_{2}\right)+K_{2} \cdot Z_{2}-b \cdot\left(\dot{Z}_{1}-\dot{Z}_{2}\right)=M_{2} \cdot g .
\end{array}\right.
$$

After some algebraic manipulation and replacing the terms that represent the shock absorber spring force, tire spring force and shock absorber damping force by $F_{K_{1}}, F_{K_{2}}$ and $F_{b}$, respectively, it is possible to obtain the following equations:

$$
\left\{\begin{array}{l}
\ddot{Z}_{1}=-\frac{\left(F_{K_{1}}+F_{b}\right)}{M_{1}}+g \\
\ddot{Z}_{2}=\frac{\left(F_{K_{1}}-F_{K_{2}}+F_{b}\right)}{M_{2}}+g .
\end{array}\right.
$$

In order to obtain the numerical solution of Eq. (6), the displacements and speeds of both reduced and "unsprung" masses were defined as state variables. The relations between this set of variables are the following: $X_{1}=Z_{1}$, $X_{2}=\dot{Z}_{1}=\dot{X}_{1}, X_{3}=Z_{2}$, and $X_{4}=\dot{Z}_{2}=\dot{X}_{3}$. Therefore, the following first order differential equations can be written (state-space form):

$$
\left\{\begin{array}{l}
\dot{X}_{1}=X_{2} \\
\dot{X}_{2}=-\frac{\left(F_{K_{1}}+F_{b}\right)}{M_{1}}+g \\
\dot{X}_{3}=X_{4} \\
\dot{X}_{4}=\frac{\left(F_{K_{1}}-F_{K_{2}}+F_{b}\right)}{M_{2}}+g .
\end{array}\right.
$$

From Eq. (7), the deflections of the shock absorber can be obtained by the difference between $X_{1}$ and $X_{3}$. The tire deflection is given directly by $X_{3}$. The other parameter of interest is the vertical load transferred to the reduced 
mass $\left(F_{v}\right)$. This force is determined by applying the D'Alembert's principle to the reduced mass as presented by the following equation:

$$
F_{v}+M_{1} \cdot \dot{X}_{2}-M_{1} \cdot g=0 .
$$

It can be observed that the vertical force $\left(F_{v}\right)$ is composed by the sum of spring and damping forces of the shock absorber, namely $F_{K_{1}}$ and $F_{b}$, respectively.

As mentioned before, in order to improve the accuracy of the mathematical model, the non-linear constitutive relations of the pneumatic spring forces $\left(F_{K_{1}}\right.$ and $\left.F_{K_{2}}\right)$ and the damping force $\left(F_{b}\right)$ are introduced in the model. Basically, the hydraulic force is considered as being proportional to the square of the deflection speed. The air compression process inside the shock absorber is based on the polytropic compression law for an ideal gas [6]. Finally, the spring characteristics of the tire are obtained from the tire manufacturer.

\subsection{Shock absorber spring force $\left(F_{K_{1}}\right)$}

The non-linear spring characteristic of the shock absorber is taken into account by considering the compression of the air and hydraulic fluid. Two springs are arranged in series and the shock absorber deflection is given by the sum of the deflections of these two springs.

As the air compression process is based on the polytropic law for the compression of gases (i.e. $P_{1} \times V_{1}^{n}=$ constant), the shock absorber pneumatic compression is given by:

$$
P_{1}=P_{0}\left(\frac{v_{0}}{v_{1}}\right)^{n}
$$

where $P_{0}$ and $P_{1}$ are the initial and instantaneous gas pressures, respectively. The ratio $v_{0} / v_{1}$ is the gas compression ratio (the initial gas volume $v_{0}$ divided by the instantaneous gas volume $v_{1}$ ) and $n$ is the polytropic coefficient. The instantaneous gas volume, $v_{1}$, is obtained by the difference between the initial gas volume, $v_{0}$, and the product of the pneumatic area, $A$, by the shock absorber deflection due to the gas compression process, $s_{1}$. By replacing the instantaneous pressure by the ratio between the spring force, $F_{K_{1}}$, and the pneumatic area, Eq. (9) can be rearranged as:

$$
F_{K_{1}}=P_{0} \cdot A \cdot\left(\frac{v_{0}}{v_{0}-A \cdot s_{1}}\right)^{n} .
$$

In the above equation, the pneumatic area corresponds to the geometrical value that is multiplied by the stroke in order to obtain the gas volume variation during the compression process. The pneumatic area can be easily calculated for the shock absorber presented in Fig. 1(b) (since the external diameter of the piston tube is known). The initial volume of the gas is based on the shock absorber internal configuration. The temperature and the process used to fill the shock absorber with oil and gas must be also taken into account for the gas volume definition. The polytropic coefficient depends both on the compression rate of the gas and the rate of heat transfer between the gas and the environment. It is known that isothermal processes have low compression rates and the polytropic coefficient is approximately 1.0 for these cases. For high compressions rates, it is expected higher values for the polytropic coefficient: the maximum value is limited to 1.4 (adiabatic process).

The hydraulic fluid stiffness, represented by the bulk modulus, corresponds to the capability of a fluid to resist a volumetric reduction due to pressure increase [7]. The rapid fluid compression with small heat transfer and large pressure excursion requires the use of an adiabatic secant bulk modulus, $\beta_{S}$. It is defined as the ratio between the pressure variation, $\Delta P$, and the total change in volume, $\Delta V$, multiplied by the initial fluid volume, $V_{0}$, as presented by the following equation:

$$
\beta_{S}=V_{0} \frac{\Delta P}{\Delta V} .
$$

The total change in the fluid volume can be expressed in terms of a shock absorber stroke, $s_{2}$, multiplied by the pneumatic area. Considering the fluid with uniform mass density and constant adiabatic secant bulk modulus, the shock absorber spring force $\left(F_{K_{1}}\right)$ can be also obtained as a function of the hydraulic fluid compression, as given below: 


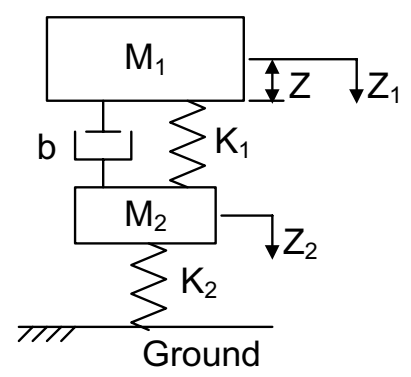

(a) Landing gear graphical representation.

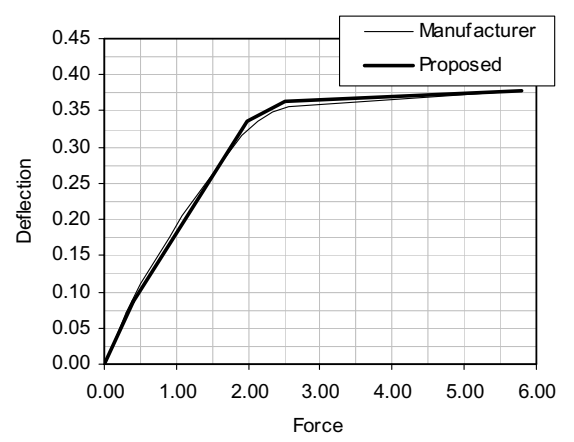

(c) Tire load vs. deflection curve.

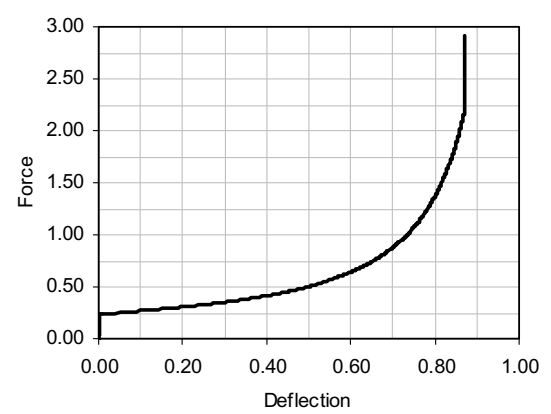

(b) Shock absorber force vs. deflection curve.

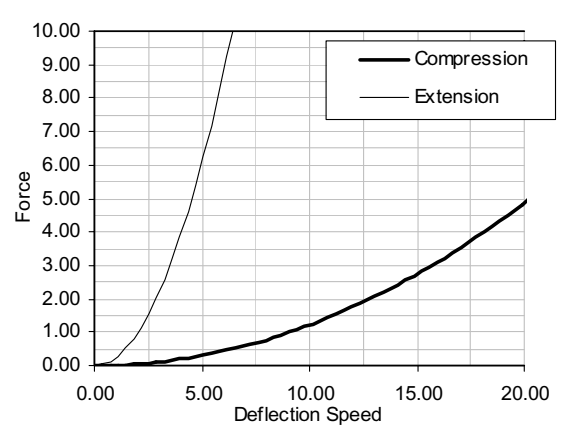

(d) Hydraulic force vs. deflection speed.

Fig. 2. Mathematical model representation.

$$
F_{K_{1}}=\frac{\beta \cdot A^{2} \cdot s_{2}}{V_{0}} .
$$

The sum of the deflections obtained from air and fluid compressions, $s_{1}$ and $s_{2}$, respectively, corresponds to the difference between the reduced and "unsprung" masses displacements, i.e., the shock absorber net deflection. Therefore, the shock absorber spring force is obtained by combing Eqs (10) and (12) in an iterative process. At each step of the dynamic simulation, the force $\left(F_{K_{1}}\right)$ is defined when the sum $s_{1}$ plus $s_{2}$ equals the difference between the displacements $Z_{1}$ and $Z_{3}$.

Figure 2(b) illustrates the compression force versus the shock absorber deflection curve. The initial force, for a zero deflection, occurs due to gas inflation pressure that represents the shock absorber spring pre-load. During the impact, the compression process starts when the pre-load value is overcome by the total force transferred to the landing gear. The gas and oil compression is governed by the previous Eqs (10) and (12). It is not the objective of the present model to detail the shock absorber stop.

\subsection{Shock absorber damping force $\left(F_{b}\right)$}

The shock absorber damping force results from the difference of pressure associated with the fluid flow through the orifices. For the considered landing gear, fully turbulent oil flow through the orifices is assumed since the relation between the diameter of the piston tube and the diameters of the orifices is enough to produce high Reynolds' number. Therefore, the equation for the fluid discharge through an orifice may be equaled to the volumetric rate of the hydraulic fluid as presented below:

$$
C_{d} \cdot A_{n} \cdot \sqrt{\frac{2}{\rho} \cdot\left(p_{h}-p_{a}\right)}=A_{h} \cdot \dot{Z},
$$




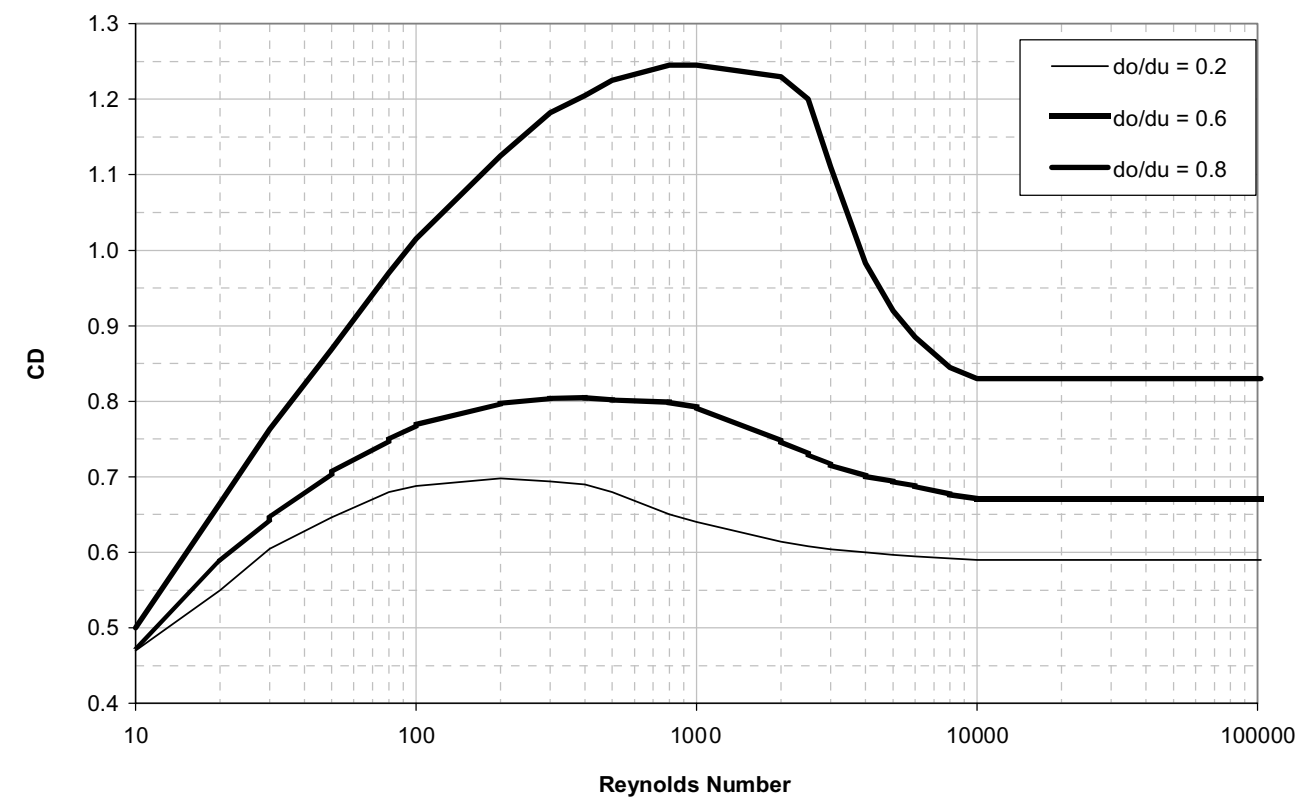

Fig. 3. Reynolds' number vs. discharge coefficient curve.

where,

$-C_{d}$ is the coefficient of discharge,

- $A_{n}$ is the net area of the orifices,

$-p_{h}-p_{a}$ is the pressure difference across the orifices,

$-\rho$ is the mass density of the hydraulic fluid,

- $A_{h}$ is the hydraulic area, and

- $\dot{Z}$ is the shock absorber deflection velocity.

Neglecting the deficiency of flow due to fluid compressibility, the hydraulic area of the shock absorber is the internal area of the piston tube cross section. The product of the discharge coefficient by the orifice area corresponds to the vena contract area that is the smallest area of the hydraulic flow when it passes through the orifice. For orifices mounted in parallel with the same discharge coefficients, the sum of the orifice areas correspond to the total net area An. The discharge coefficient depends on several factors, such as the variations of both upstream and downstream flow chambers, and the variation of the Reynolds' number during the compression of the shock absorber.

From Eq. (13), the pressure difference across the orifices can be expressed as:

$$
p_{h}-p_{a}=\frac{\rho \cdot A_{h}^{2} \cdot \dot{Z}^{2}}{2 \cdot\left(C_{d} \cdot A_{n}\right)^{2}} .
$$

As mentioned before, the shock absorber damping force results from the difference of pressure across the orifices multiplied by the hydraulic area (area subjected to hydraulic pressure). Therefore, from the above equation, the shock absorber damping force $\left(F_{b}\right)$ is determined by the product between the damping parameter, $D S$, and the deflection velocity squared, as presented in the following equation:

$$
F_{b}=\frac{\rho \cdot A_{h}^{3} \cdot \dot{Z}^{2}}{2 \cdot\left(C_{d} \cdot A_{n}\right)^{2}}=\frac{\dot{Z}}{|\dot{Z}|} \cdot D S \cdot g \cdot \dot{Z}^{2},
$$

where $D S=\frac{\rho \cdot A_{h}^{3}}{2 \cdot g \cdot\left(C_{d} \cdot A_{n}\right)^{2}}$ is a constant value.

Because of the sensitivity of the discharge coefficient with respect to the Reynolds' number variation and considering the severe variation of the flow rates in the shock absorber compression process, it is difficult to obtain an 
accurate representation of $C_{d}$. In [7], the behavior of the discharge coefficient due to the Reynolds' number variation is related with the ratio between the orifices diameter and the upstream area $\left(d_{0} / d_{u}\right)$ as shown in Fig. 3. As can be observed, for high Reynolds' number, the discharge coefficient assumes a constant value. Therefore, for the landing gear compression, where a turbulent flow with high Reynolds' number is expected, it is reasonable using a constant value for $C_{d}$ and, consequently, for the damping parameter.

The resulting curves for the shock absorber compression and extension phases are presented in Fig. 2(d). They are obtained by using the quadratic relation between the hydraulic force and the shock absorber deflection speed as given above, and by considering that the number of orifices reduces along of the shock absorber extension to avoid rebound effect. The damping parameter for the shock absorber extension is not the object of the present study, since no relevant loads are transferred to the reduced mass at this stage.

\subsection{Tire spring force $\left(F_{K_{2}}\right)$}

Understanding the characteristics of the tire interaction with the ground is essential for studying the dynamic loads involved in drop tests. Basically, the radial stiffness and stroke of the tire must be sufficient to absorb the vertical loads transferred to the landing gear without bottoming. How much the tire participates on the process of energy absorption depends on the shock absorber characteristics such as inflating pressure and compression rate.

For the present study it is required to know the behavior of the tire deflection with respect to the vertical load variation for a particular tire inflating pressure. The non-linear characteristics and the tire bottoming must be also taken into account. These tire properties are represented by the dynamic load versus deflection curve as supplied by the tire manufacturer (see in Fig. 2c). The same curve is used for the compression and expansion of the tire. Therefore, the tire is modeled as a pure spring element, neglecting the hysteretic effect.

Regarding the mathematical model, the tire load versus deflection curve is divided according to four linear regions aiming at applying the information found in graphs provided by the tire manufacturer. Therefore, the tire stiffness assumes four different values depending on the tire deflection region at each simulation step. Regarding the first and second regions of the load versus deflection curve, a slightly difference in the tire stiffness is observed. In the third region, the tire bottoming process starts. From a practical point of view, the maximum landing load for a particular landing gear must not exceed the third region of the load versus deflection curve. The last region depicts the tire bottoming, where a severe increase of the force as a function of the deflection is observed. A comparison between the proposed force versus deflection curve and the one obtained from the tire manufacturer is presented in Fig. 2(c).

\section{Numerical optimization as an identification tool}

Numerical optimization techniques have been widely used, since they take advantage of computer automation capabilities through a set of mathematical methods that operate according to the formulation [8]:

$$
\underset{\mathbf{x}}{\operatorname{Minimize}} F(\mathbf{x})
$$

subject to:

$$
\left\{\begin{array}{l}
g_{j}(\mathbf{x}) \leqslant 0, \mathrm{j}=1,2,3, \ldots, \mathrm{M} \\
h_{k}(\mathbf{x})=0, \mathrm{k}=\mathrm{M}+1, \mathrm{M}+2, \mathrm{M}+3, \ldots, \mathrm{L} ; \\
\mathbf{x}_{i}^{L} \leqslant \mathbf{x}_{i} \leqslant \mathbf{x}_{i}^{U}, \mathrm{i}=1,2,3, \ldots, \mathrm{N}
\end{array}\right.
$$

where $F(\mathbf{x})$ is the objective function, $g_{j}(\mathbf{x})$ and $h_{k}(\mathbf{x})$ are the inequality and equality constraints, respectively, and $\mathbf{x}_{i}^{L} \leqslant \mathbf{x}_{i} \leqslant \mathbf{x}_{i}^{U}$ imposes side constraints to the design space.

In summary, the optimization problem is compound by the design space (or search space), where all possible solutions for a problem are considered; the objective function (known also as cost function, fitness function, evaluating function), that is the way to evaluate each point of the design space; the constraints, that impose restrictions to the project; and the solver, or in the other words, the algorithm that will look for the solution of the optimization problem. As a consequence, the key of the success of this work resides in the choice of the appropriate method that will best manage to the solution of the problem. In this sense, the solver can be implemented by using a classical method as 


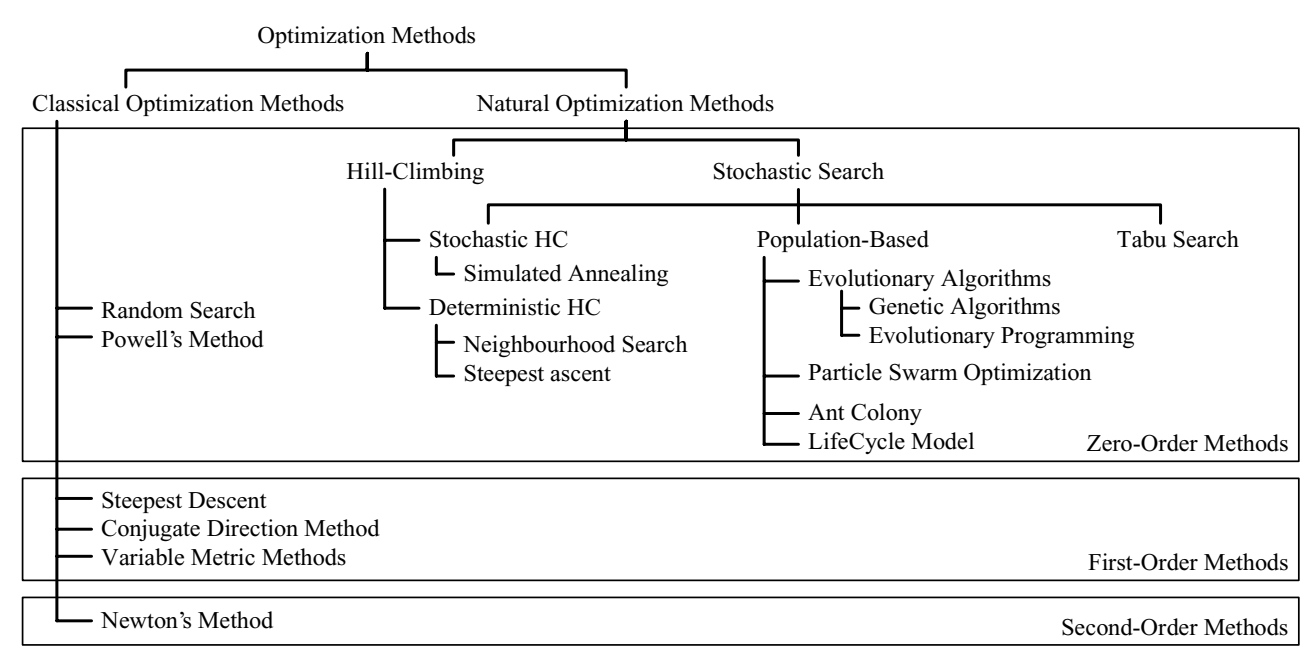

Fig. 4. Optimization methods (adapted from [10]).

based on differential calculus [8], or alternatively, the algorithm can be motivated by some natural phenomena [9, 10]. Figure 4 shows a classification scheme including various optimization approaches that can be classified as either classical or nature-inspired.

It is worth mentioning that in the context of system identification, the objective function must express how different a "trial system", characterized by a set of trial parameters, and the system to be identified are. In other words, the objective function makes use of a set of measures that describe the behavior of the system and the numerical counterparts to build a functional, which when minimized gives a possible solution for the identification problem.

\section{Nature-inspired optimization}

\subsection{Genetic algorithms}

GA is an optimization algorithm used to find approximate solutions to difficult-to-solve problems through application of the principles of evolutionary biology to computer science. GA is based on Darwin's theory of survival and evolution of species, as explained in [9,11]. GA uses biologically-derived concepts such as inheritance, mutation, natural selection, and recombination (or crossover).

The algorithm starts from a population of random individuals, viewed as candidate solutions to the problem. During the evolutionary process, each individual of the population is evaluated, reflecting its adaptation capability to the environment. Some of the individuals of the population are preserved while others are discarded; this process mimics the natural selection in the Darwinism. The remaining group of individuals is paired in order to generate new individuals to replace the worst ones in the population, which are discarded in the selection process. Finally, some of them can be submitted to mutation, and as a consequence, the chromosomes of these individuals are altered. The entire process is repeated until a satisfactory solution is found. The outline of a basic GA is as shown in Fig. 5.

Although the initially proposed GA algorithm was dedicated to discrete variables only, nowadays improvements are available to deal with discrete and continuous variables. Details about GA are provided in [9,11].

\subsection{Particle swarm optimization}

PSO was introduced by Kennedy and Eberhart in [12]. The method reflects the social behavior of some bird species. The main idea of the PSO is to reproduce the search procedure used by a swarm of birds when looking for food or nest. Consider the following situation: a swarm of birds is searching for food around a delimited area. Suppose there is just one place where food can be found and the birds do not know where it is, then if one bird is 


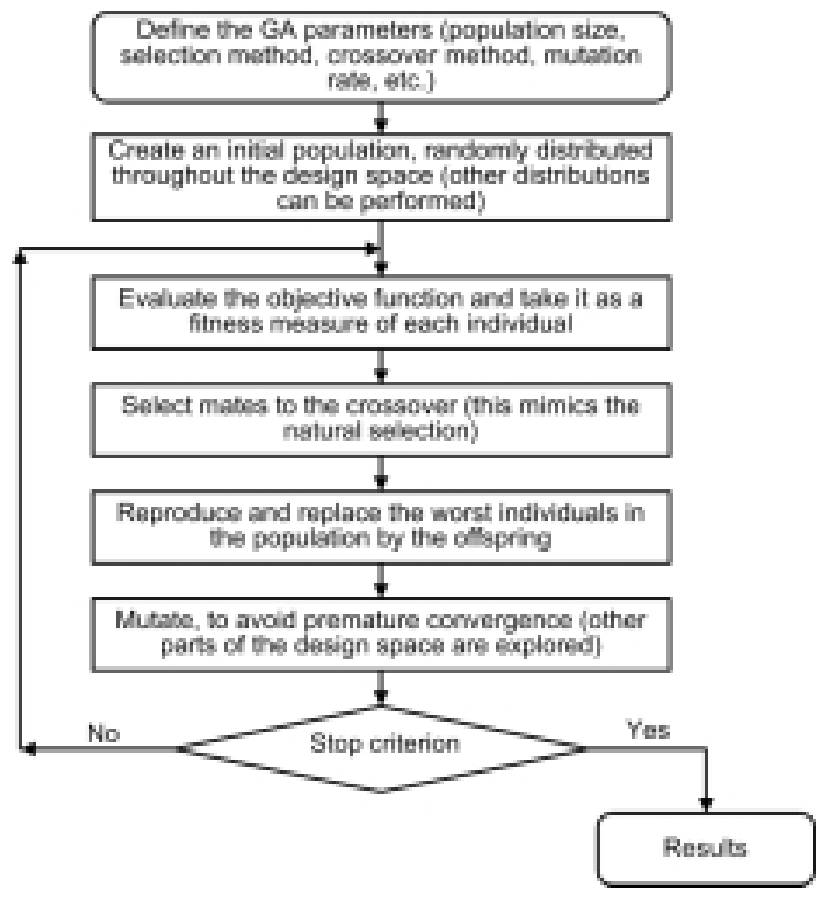

Fig. 5. GA basic algorithm.

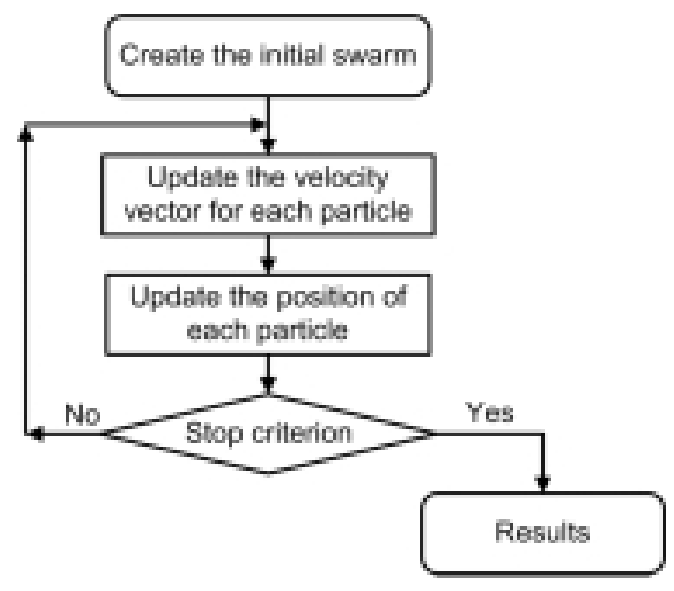

Fig. 6. PSO basic algorithm.

successful in its search, it can attract other birds, and as a result of this social behavior, the others will find the food too. Following this principle, each individual learns (and contributes) primarily to the success of his neighbors. This fact requires the balance between exploration (the capacity of individual search) and exploitation (the capacity of learning from the neighbors).

Behind of the scenes, this is achieved by modeling the flying using a velocity vector, somewhat analogous to a search direction. The velocity vector considers a contribution of the current velocity and other two portions referred to the knowledge of the particle itself and of the swarm, respectively, about the search space. In such a way, the velocity vector is used to update the position of each particle in the swarm. The outline of a basic PSO algorithm is given by Fig. 6. PSO is comprehensively presented by Kennedy and Eberhart in [12] and Venter and Sobieski in [13]. 


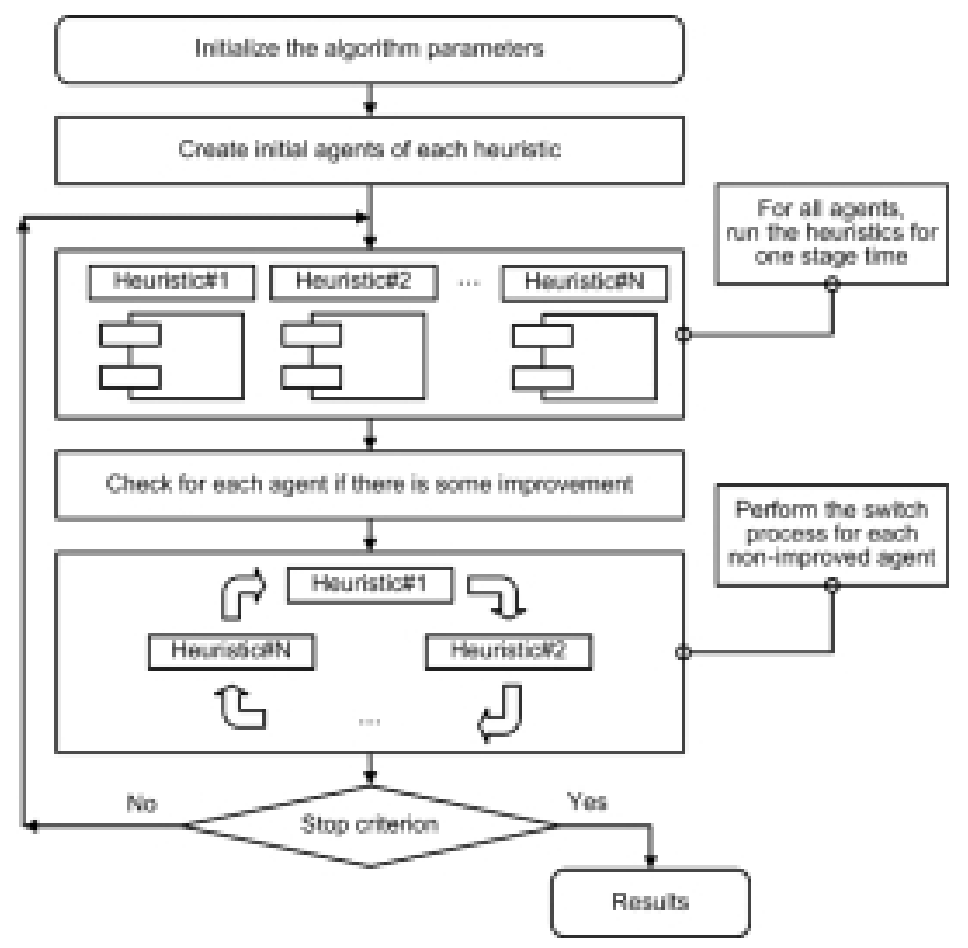

Fig. 7. LifeCycle basic algorithm.

\subsection{LifeCycle model}

LC is a hybrid nature-inspired optimization method inspired by the idea of life cycle stages, initially proposed by Krink and Løvberg in [3]. From the mathematical point of view, natural algorithms such as GA and PSO are heuristics search methods of proven efficiency as optimization tools. LC intends to put together the positive characteristics found in each method and creates a self-adaptive optimization approach. Each individual, as a candidate solution, decides based on its success if it would prefer to belong to a population of a GA or to a swarm of a PSO. This means that various heuristic techniques contribute together to form a robust high performance optimization tool. The idea is that complex problems can be conveniently considered from the optimization viewpoint. As can be viewed, the less well-succeeded individuals must change their status in order to improve their fitness. This means that the optimization approach does not follow a rigid scheme, in which various techniques are used sequentially in a cascade-type structure. In other words, it is the mechanism of self-adaptation of the optimization problem that rules the procedure. An outline of LC is presented in Fig. 7.

Since the algorithm is composed by various heuristics, it is necessary to set the parameters of every heuristic used in the LC. Nevertheless, there is a parameter inherent to the LC, namely the number of iterations that represents a stage of the LC, called as stage interval. At the end of each stage interval, the less well-succeeded individuals must change their stage in order to improve their fitness. During the optimization procedure the agents of each subpopulation commute to the other in such a way to improve its own fitness. In other words, it is the mechanism of self-adaptation of the optimization problem that rules the procedure. It means that LC does not follow a rigid cascade-scheme in which different algorithms are used in sequence with the results of one being used as initial configuration to the other (one illustration of this strategy can be viewed in [14]). To close the definition, LC stages must be presented. In the present work, two heuristics are used as stages, namely the GA and the PSO. Other versions of the LC can be proposed by considering other heuristics and a mix of them, as shown in [3]. For more complete information about LC Model check [3,15].

The SIMPLE Optimization Toolbox [16], a toolbox developed at Federal University of Uberl ândia as an add-on to MATLAB ${ }^{\circledR}$, provides implementation for all solvers used to deal with the optimization problem. 


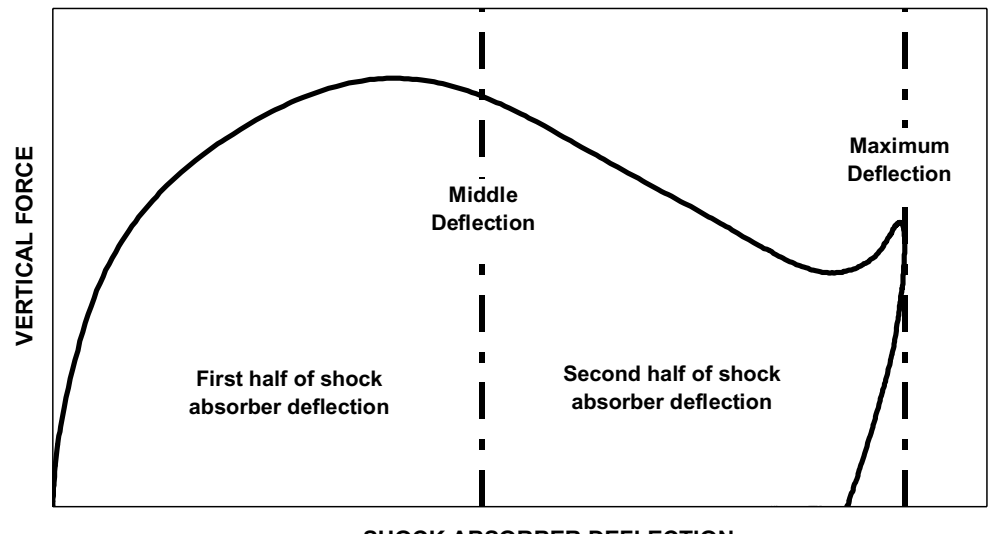

SHOCK ABSORBER DEFLECTION

Fig. 8. Vertical force vs. deflection curve.

\section{Identification written as an optimization problem}

The dynamic behavior of the landing gear is defined in the initial phase of the design by using a proposed mathematical model that has some parameters not so accurately predicted, such as: damping parameters for the shock absorber compression and extension phases, polytropic coefficient, "unsprung" mass, etc. In the initial phase of the landing gear design, it is common to use theoretical values for these parameters. From the drop test results, an identification methodology is applied in order to adjust the theoretical parameters of the landing gear mathematical model to the experimental ones. Depending on the applicability of the landing gear model, some of these parameters have more important features in the system dynamic behavior than others.

As previously mentioned, the main focus of the proposed mathematical model is to predict the maximum vertical loads transferred to the reduced mass and the maximum deflections of tire and shock absorber. In this context, some parameters may be initially neglected in the identification process. For example, the damping parameter for the shock absorber extension is an unknown parameter that has important functions to avoid the rebound effect following the landing impact and to improve the aircraft handling characteristics, however this parameter does not affect the magnitude of the maximum loads and deflections that the model intents to predict. Therefore, it is not mandatory to identify this parameter for the present model.

The parameters selected for identification in the contest of the present contribution are the following: the polytrophic coefficient, $n$, and the damping parameter for shock absorber compression, $D S$. Therefore, the corresponding identification problem consists in finding the set of design variables $\mathbf{x}=[n D S]^{T}$ that minimizes the multi-objective function as given by:

$$
\begin{aligned}
J(\mathbf{x})= & \frac{\left|X_{1 \text { trial }}^{\max }-X_{1 \exp }^{\max }\right|}{X_{1 \exp }^{\max }}+\frac{\left|X_{3 \text { trial }}^{\max }-X_{3 \exp }^{\max }\right|}{X_{3 \exp }^{\max }}+\frac{\left|Z_{\text {trial }}^{\max }-Z_{\exp }^{\max }\right|}{Z_{\exp }^{\max }} \\
& +\frac{\left|F_{V_{1} \text { trial }}^{\max }-F_{V_{1} \exp }^{\max }\right|}{F_{V_{1} \exp }^{\max }}+\frac{\left|F_{V_{2} \text { trial }}^{\max }-F_{V_{2} \exp }^{\max }\right|}{F_{V_{2} \exp }^{\max }}
\end{aligned}
$$

where $X_{1}$ is the vertical displacement of the reduced mass, $X_{3}$ is the tire deflection, $Z$ is the shock absorber deflection, and $F_{V_{1}}$ and $F_{V_{2}}$ are the values of the maximum vertical forces. As can be seen from the above formula, a weight of 1.0 is used to ponder all the individual objective functions.

$F_{V_{1}}$ is the vertical force that corresponds to the first half of the shock absorber deflection stage. $F_{V_{2}}$ is the vertical force that corresponds to the second half of the deflection stage. Figure 8 illustrates the behavior of both $F_{V_{1}}$ and $F_{V_{2}}$.

The polytropic coefficient ranges between 1.0 and 1.4 for isothermal and adiabatic processes, respectively. The discharge coefficient was theoretically calculated by taking into account the diameters of the orifices of the shock 
Table 1

Parameter setup for LC in the landing gear application

\begin{tabular}{|c|c|c|c|}
\hline Number of individuals & \multicolumn{2}{|c|}{ Number of iterations } & Stage interva \\
\hline \multicolumn{2}{|l|}{10} & 50 & 5 \\
\hline \multicolumn{4}{|c|}{ Table 2} \\
\hline \multicolumn{4}{|c|}{ Experimental and simulated results for the landing gear } \\
\hline Parameter & Drop test & Simulation & Error $(\%)$ \\
\hline$F_{V_{1}}^{\max }(\mathrm{N})$ & $56,650.4$ & $57,781.0$ & 2.0 \\
\hline$F_{V_{2}}^{\max _{2}}(\mathrm{~N})$ & $65,212.3$ & $63,278.5$ & -3.0 \\
\hline$X_{1}(\mathrm{~m})$ & 0.315 & 0.318 & 1.2 \\
\hline$X_{3}(\mathrm{~m})$ & 0.088 & 0.082 & -6.1 \\
\hline$Z(\mathrm{~m})$ & 0.229 & 0.231 & 1.1 \\
\hline
\end{tabular}

absorber and the Reynolds' number for the upstream flow condition. The bounds of the search space for the damping parameter were arbitrarily defined as $\pm 15 \%$ of the theoretical value $D S_{T H}$.

The model updating process has been historically very time consuming, i.e., many iterations are often needed before obtaining a good match between experimental testing and analytical results. By using the optimization process (which can also be seen as an automated procedure) presented in this work, a reduction of engineering time as large as $50 \%$ to $60 \%$ for each drop test run has been obtained. The computation time necessary to solve the optimization problem was approximately 50 minutes. These results were obtained by using a PC - Pentium $1.6 \mathrm{GHz}$ processor, 256 MB, running Windows XP and MATLAB 6.5.0 Release 13.

In future work, other optimization techniques will be tested for comparison purposes, involving both computation time and accuracy of results.

\section{Identification results}

Table 1 shows the setup for LC that was used to handle the present problem. The multi-objective function was minimized for the following values:

- Polytropic coefficient: 1.05 .

- Damping parameter: $622 \mathrm{~kg} . \mathrm{s}^{2} / \mathrm{m}^{2}$.

Currey in [1] recommended using a polytropic coefficient of 1.1 for shock absorbers without separator piston between gas and oil. Since this value is used only as reference and some variations can occur, for the studied shock absorber, which has oil in contact with the gas, the obtained value of 1.05 is considered as a good approach. The obtained damping parameter is approximately $+9 \%$ above of the theoretical value, which represents also a good matching.

Figure 9 shows the graphics obtained for the simulations with the identified parameters and the drop test cases. Table 2 compares some experimental and simulated quantities. Again, the good performance and robustness of LifeCycle together with the proposed mathematical model were demonstrated. As can be seen, the agreement between experimental and simulated curves is satisfactory. The magnitude of the obtained loads variation is acceptable for a landing gear in the context of airplane design.

Specifically for the tire deflection and vertical force, given by $X_{3}$ and $F_{v}$, respectively, small differences between experimental and theoretical results are found. This is mainly due to the flexibility effect of the landing gear fork that was not considered in the proposed mathematical model.

\section{Conclusion}

This work was dedicated to the application of a nature-inspired optimization technique to solve the inverse problem represented by the identification of an aircraft landing gear model. The model was described in terms of the landing gear geometry, internal volumes and areas, shock absorber travel, tire type, and gas and oil characteristics of the 


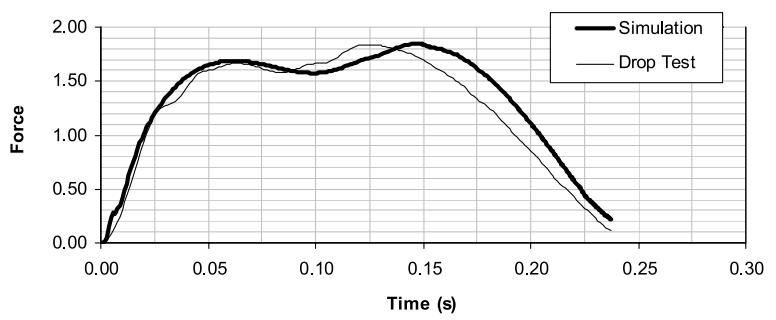

(a) Reduced mass - vertical force $\left(F_{v}\right)$.

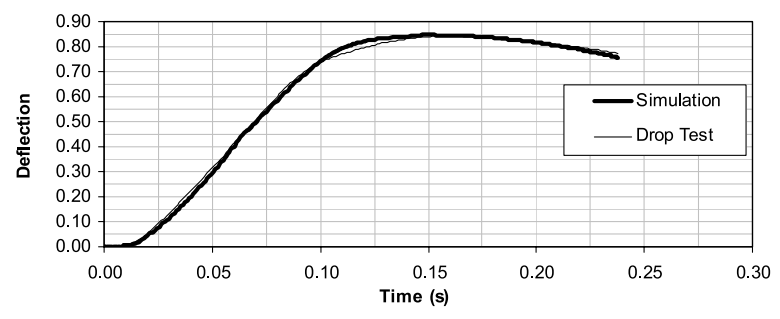

(c) Shock absorber deflection $(Z)$.

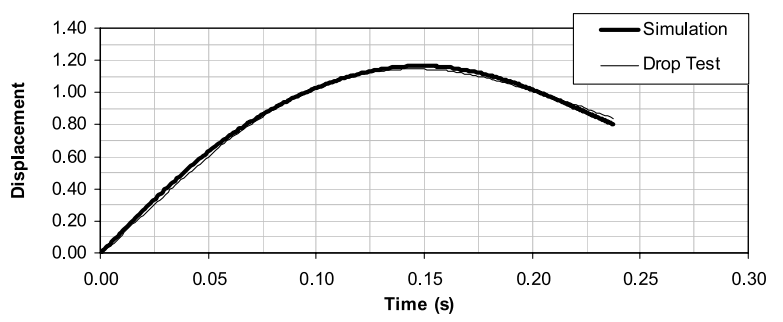

(b) Reduced mass - vertical displacement $\left(X_{1}\right)$.

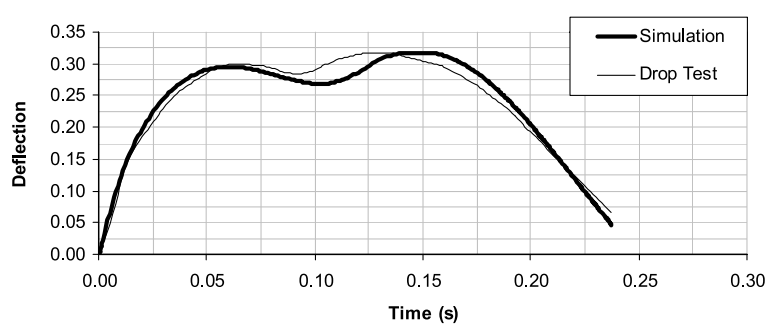

(d) Tire deflection $\left(X_{3}\right)$.

Fig. 9. Comparison between simulation and experimental data.

pneumatic shock absorber. The solution to this inverse problem was obtained by using a nature-inspired approach based on a method known as LifeCycle Model.

It was shown how optimization techniques can be used in the context of inverse problems. For this aim, it was shown that the multi-objective function makes use both of a set of measures that describe the behavior of the system and its numerical counterparts to build a representative functional, which once minimized gives a possible solution for the identification problem. Two nature-inspired optimization tools, namely the Genetic Algorithms and Particle Swarm Optimization were briefly revisited. Then, the LifeCycle, a hybrid nature-based optimization method inspired by the idea of life cycle stages, was introduced. Then, it was shown how LC puts GA and PSO together to create a self-adaptive optimization approach.

Finally, the problem of landing gear parameter identification was addressed. The behavior of the landing gear was simulated as a non-linear model. It was found a good match between the theoretical results and those obtained from the experimental drop-test. Therefore, it can be concluded that the proposed identification approach was proved successful thus indicating that the methodology can be used in more complex models, such as the ones in which wheel rotation for the representation of drag loads and landing gear flexibility are included (in addition to the two degrees of freedom considered in the present model).

It is important to add that the landing loads requirements for transport airplanes have been updated in the last years as a consequence of the increasing complexity of the landing gear shock absorber systems and airplane structures. Recently, the FAA (Federal Aviation Administration) has revised the shock absorption test requirements for transport aircrafts where the complete validation of the landing gear dynamic characteristics is required. In this context, it is proposed for a future work to adequate the landing gear model presented in the present contribution to the recent requirements of the shock absorption tests by including the wheel rotation and the longitudinal degree of freedom of the landing gear leg. With this approach, the wheel axle longitudinal force produced due to interaction between tire and ground will be represented by the mathematical model. Optimization techniques will be used to adjust the simulated longitudinal forces with those obtained in the free drop tests. From the optimization point of view, parameters as slip ratio curve (tire to ground friction versus tire slip), structural stiffness, structural damping and effective moment of inertia of wheel and tire assembly will be used as design variables in order to minimize the cost function. With the identified parameters and the landing gear mathematical model validated, it will be possible to predict the landing gear dynamic response due to changes on tires (manufacturer, ply rating, inflating pressure, etc.), airplane weight, shock absorber inflating pressure, etc., without the necessity of free drop tests. 


\section{Acknowledgements}

Mr. Zanini and Mr. Magalhães, they both are grateful to Embraer. Dr. Góes thanks ITA and Embraer. Msc. Felipe Viana is thankful to CNPq Brazilian Research Agency for his PhD scholarship and Dr. Valder Steffen, Jr acknowledges CNPq for the partial financing of the present research work through Proc. 470346/2006-0.

\section{References}

[1] N.S. Currey, Landing Gear Design Handbook, Published by the Lockheed-Georgia Company, USA, 1984.

[2] N.S. Currey, Aircraft Landing Gear Design: Principles and Practices, Published by AIAA, USA, 1988.

[3] T. Krink and M. Løvberg, The LifeCycle Model: Combining Particle Swarm Optimisation, Genetic Algorithms and Hill-Climbers, in Proceedings of the 7th International Conference on Parallel Problem Solving from Nature, 2002, 621-630.

[4] K. Kristinsson and G.A. Dumont, System identification and control using genetic algorithm, IEEE Trans Syst, Man, Cybern 22(5) (1992), 1033-1046.

[5] J.E. Rojas, F.A.C. Viana, D.A. Rade and V. Steffen Jr., Force Identification of Mechanical Systems by Using Particle Swarm Optimization, in: 10th AIAA/ISSMO Multidisciplinary Analysis and Optimization Conference, Albany, 2004.

[6] B. Milwitzky and F.E. Cook, Analysis of Landing Gear Behavior, NACA Report 1154, 1954.

[7] G.R. Keller, Hydraulic System Analysis, Published by the Editors of Hydraulics \& Pneumatics Magazine, USA, 1974.

[8] G.N. Vanderplaats, Numerical Optimization Techniques for Engineering Design, Vanderplaats Research and Development, Inc., 4rd ed, 2005.

[9] L.R. Haupt and S.E. Haupt, Practical Genetic Algorithms, Wiley-Interscience Publication, New York, USA, 1998.

[10] T.K. Rasmussen, Improving Particle Swarm Optimization by hybridization of stochastic search heuristics and Self-Organized Criticality, Doctoral thesis, University of Aarhus, Department of Computer Science, Aarhus C, Denmark, 2002.

[11] Z. Michalewicz, Genetic Algorithms + Data Structures = Evolutionary Programs, 2nd edition, Springer-Verlag, New York, USA, 1994.

[12] J. Kennedy and R.C. Eberhart, Particle Swarm Optimization, Proceedings of the 1995 IEEE International Conference on Neural Networks, Perth, Australia, 1995, 1942-1948.

[13] G. Venter and J.S. Sobieski, Particle Swarm Optimization, Proceedings of the 43rd AIAA/ASME/ASCE/AHS/ASC Structures, Structural Dynamics, and Materials Conference, Vol. AIAA 2002-1235, Denver, CO - USA, 2002.

[14] E.G. Assis and V. Steffen Jr., Inverse Problem Techniques for the Identification of Rotor-Bearing Systems, Inverse Problems in Engineering 11(1) (2003), 39-53.

[15] J.E. Rojas, F.A.C. Viana, D.A. Rade and V. Steffen Jr., Identification of External Loads in Mechanical Systems Through Heuristic-based Optimization Methods and Dynamic Responses, Latin American Journal of Solids and Structures (2004).

[16] F.A.C. Viana and V. Steffen Jr., SIMPLE Optimization ToolBox - Users Guide, 2th edition, http://www.geocities.com/fchegury/, [downloaded at November 2006] 

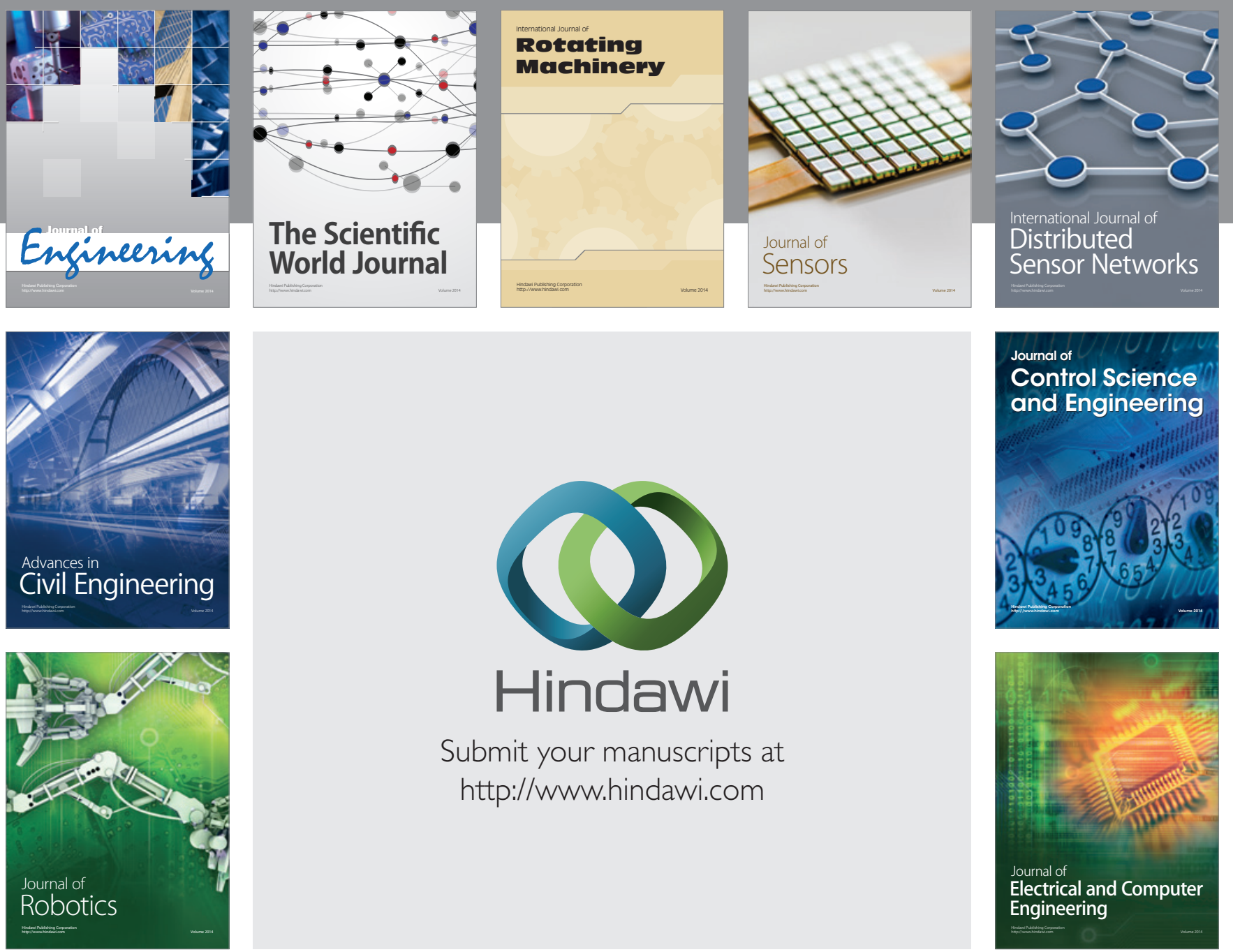

Submit your manuscripts at

http://www.hindawi.com
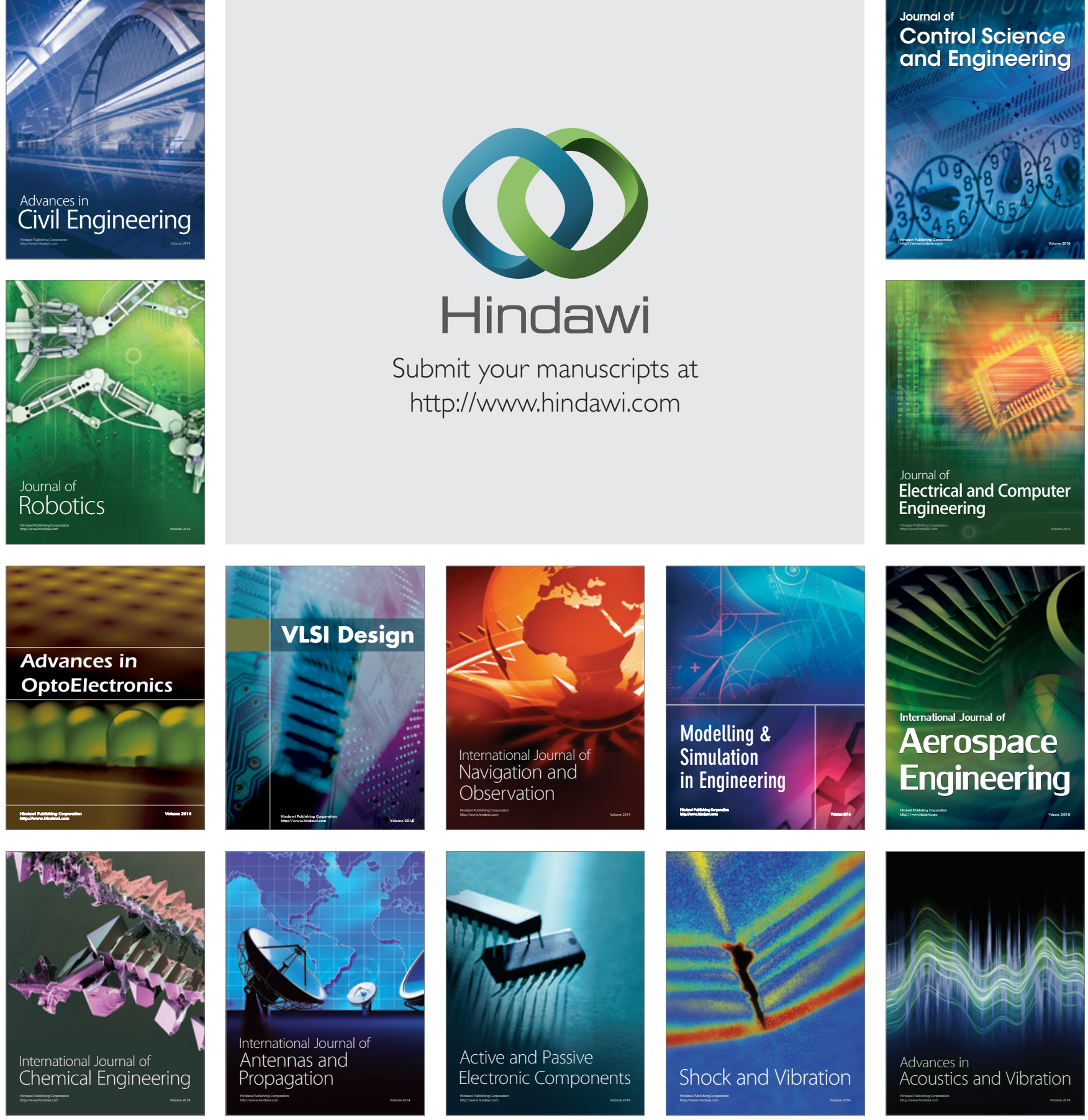